Check for updates

Cite this: RSC Adv., 2018, 8, 15567

\title{
Silver nanoparticle-loaded microgel-based etalons for $\mathrm{H}_{2} \mathrm{O}_{2}$ sensing $\dagger$
}

Received 13th March 2018

Accepted 16th April 2018

DOI: $10.1039 / \mathrm{c} 8 \mathrm{ra02215a}$

rsc.li/rsc-advances

\author{
Tong Shu, ab Qiming Shen, ${ }^{\text {b }}$ Yu Wan, ${ }^{\text {b }}$ Wei Zhang, ${ }^{\text {b }}$ Lei Su, ${ }^{a}$ Xueji Zhang ${ }^{a}$ \\ and Michael J. Serpe (ID*b
}

Silver nanoparticles (AgNPs) were generated inside the network structure of poly( $N$-isopropylacrylamide)co-acrylic acid (pNIPAm-co-AAc) microgels that were sandwiched between two thin Au layers (15 nm) of an etalon. This was done by introducing $\mathrm{Ag}^{+}$to the etalons composed of deprotonated microgels, followed by its subsequent reduction with $\mathrm{NaBH}_{4}$. The resultant microgels were collected and then characterized by transmission electron microscopy (TEM), Fourier transform infrared spectroscopy (FTIR) and X-ray photoelectron spectroscopy (XPS), verifying the loading of AgNPs with relatively uniform diameter (5-7 $\mathrm{nm}$ ) within the microgels. Furthermore, the optical properties of the resultant etalons and their response to $\mathrm{H}_{2} \mathrm{O}_{2}$ were evaluated by reflectance spectroscopy. Specifically, upon the addition of $\mathrm{H}_{2} \mathrm{O}_{2}$, the AgNPloaded etalons exhibited both a red shift in the position of the reflectance peaks and an increase in reflected wavelength intensity. We hypothesize that the dual signal response of the devices was a result of oxidative decomposition of the AgNPs, enabling the microgels to swell and for more light to be reflected (due to the loss of the light absorbing AgNPs). Finally, we showed that the AgNPs could be regenerated in the used etalons multiple times without a loss in performance. This work provides a costeffective means to detect $\mathrm{H}_{2} \mathrm{O}_{2}$, which could be modified to sense a variety of other species of physiological and environmental importance through rationally loading other functional nanomaterials.

\section{Introduction}

Photonic crystals have attracted considerable interest due to their low cost, ease of signal readout, simple operation, and their ability to be modified to detect multiple analytes..$^{1-4}$ The Serpe Group pioneered the development of poly $(\mathrm{N}$-isopropylacrylamide) (pNIPAm) microgel-based etalons in 2010., Since then, they have been shown to exhibit visual color that can be made to depend on the presence of certain species, or the state of a system, e.g. , temperature, $\mathrm{pH},{ }^{7} \mathrm{Cu}^{2+},{ }^{8} \mathrm{CO}_{2},{ }^{9} \mathrm{H}_{2} \mathrm{O}_{2},{ }^{4}$ proteins, ${ }^{10}$ and DNA. ${ }^{11}$ Typically, etalons are constructed by sandwiching a layer of pNIPAm microgels between two thin Au layers. ${ }^{12}$ At a fixed observation angle, the position of the peaks in a reflectance spectrum (and hence the device color) depends primarily on the distance between the two gold layers. ${ }^{6}$ The ability of the etalons to change color is derived from the responsivity of the microgel layer. Importantly, modification of microgels with functional groups, e.g., 4-vinylpyridine, can allow the etalons to respond to specific species. ${ }^{9}$

\footnotetext{
${ }^{a}$ Beijing Key Laboratory for Bioengineering and Sensing Technology, Research Center for Bioengineering and Sensing Technology, School of Chemistry and Biological Engineering, University of Science and Technology Beijing, Beijing 100083, P. R. China ${ }^{b}$ Department of Chemistry, University of Alberta, Edmonton, Alberta, Canada T6G 2G2. E-mail: michael.serpe@ualberta.ca; Fax: +1 780492 8231; Tel: +1 7804925778 $\dagger$ Electronic supplementary information (ESI) available. See DOI: $10.1039 / \mathrm{c} 8 \mathrm{ra} 02215 \mathrm{a}$
}

The past three decades have witnessed an incredible increase in the development of nanomaterials, ${ }^{13-16}$ due to their remarkable size- and shape-dependent electronic, magnetic and optical properties (i.e., the quantum confinement effect) as well as their ability to be used in various applications, e.g., sensors. ${ }^{17,18}$ To generate nanoparticles with narrow size distributions, ligands that yield repulsive forces between nanoparticles to control aggregation are often used. ${ }^{19}$ Of importance for this investigation are pNIPAm-based microgels, which have emerged as a novel and promising nanoparticle stabilizer. ${ }^{20}$ That is, the interior of microgels can serve as a microreactor for generating nanoparticles. For instance, Shi et al. synthesized $\mathrm{Au}$ nanoparticles inside pNIPAm-co-methacrylic acid microgels via an in situ reduction reaction. ${ }^{21}$ In a previous study, we showed that pNIPAm-co-acrylic acid (pNIPAm-co-AAc) microgels could be used as a scaffold for the in situ generation of silver nanoparticles (AgNPs), which could subsequently be used to detect $\mathrm{H}_{2} \mathrm{O}_{2}$ in solution. ${ }^{22}$

As a reactive oxygen species (ROS), $\mathrm{H}_{2} \mathrm{O}_{2}$ is generated from almost all biological oxidative cycle reactions. ${ }^{23} \mathrm{H}_{2} \mathrm{O}_{2}$ also plays an essential role in modulating a number of physiological processes. For instance, the homeostasis of $\mathrm{H}_{2} \mathrm{O}_{2}$ possesses significant impacts on cell proliferation, death, and signal transduction. ${ }^{24}$ On the other hand, excessive amounts of $\mathrm{H}_{2} \mathrm{O}_{2}$ in the human body can generate irreversible oxidative damage, resulting in accelerated aging, ${ }^{25}$ neurodegeneration, ${ }^{26}$ DNA 
damage,$^{27}$ and cancer. $^{28}$ Thus, it is essential to continuously develop new and improved tools to quantify $\mathrm{H}_{2} \mathrm{O}_{2}$. In this investigation, we show that microgels in etalons can be used as a scaffold for the generation and stabilization of AgNPs, which we show can be used for generating a "dual" optical response to $\mathrm{H}_{2} \mathrm{O}_{2}$. Specifically, we show that an in situ synthetic route for generating AgNP-loaded pNIPAm-co-AAc hybrid microgels in etalons could be achieved by first exposing deprotonated pNIPAm-co-AAc microgels to $\mathrm{Ag}^{+}$. The negative charges on the deprotonated AAc groups allowed the microgels to strongly interact, and become enriched, with $\mathrm{Ag}^{+}$. AgNPs with narrow size distribution could then be obtained by performing the reduction at low temperature. The resultant AgNP-loaded etalons were shown to exhibit color due to the structure of the etalon and the absorbance of light by the AgNPs. We hypothesize that the color of the devices changed upon addition of $\mathrm{H}_{2} \mathrm{O}_{2}$ due to the degradation of the AgNPs, which causes a red-shift in the reflectance peaks from the etalon and an increase in the intensity of the reflectance peaks. To this end, we investigated the response of the AgNP-loaded etalon to various concentrations of $\mathrm{H}_{2} \mathrm{O}_{2}$. The dual optical signal change of the etalon can then be used to detect $\mathrm{H}_{2} \mathrm{O}_{2}$, while also providing the possibility for correcting for interferants in solution. ${ }^{5-8}$ We go on to show that the sensing performance of the AgNP-loaded etalon could be fully restored by a repeated exposure to $\mathrm{Ag}^{+}$and subsequent AgNPs generation.

\section{Experimental section}

\section{Materials and methods}

NIPAm was purchased from TCI (Portland, OR) and purified by recrystallization from hexanes (EMD, Gibbstown, OR). $N, N$ Methylenebisacrylamide (BIS), acrylic acid (AAc), ammonium persulfate (APS), silver nitrate $\left(\mathrm{AgNO}_{3}\right)$, sodium hydroxide $(\mathrm{NaOH})$, hydrogen chloride $(\mathrm{HCl})$ and sodium borohydride $\left(\mathrm{NaBH}_{4}\right)$ were purchased from Sigma-Aldrich (Oakville, ON). Microgels were lyophilized using a VirTis bench-top K-manifold Freeze Dryer (Stone Ridge, New York). Deionized (DI) water with a resistivity of $18.2 \mathrm{M} \Omega \mathrm{cm}$ obtained from a Milli-Q Plus system (Billerica, MA), was utilized in this study.

\section{Characterization}

A Hitachi H-7650 transmission electron microscope (TEM, Japan) with an accelerating voltage of $200 \mathrm{kV}$ was utilized to obtain TEM images. X-Ray Photoelectron Spectroscopy (XPS) was performed on a Kratos AXIS Ultra spectrometer equipped with a monochromated Al $\mathrm{K} \alpha(h \nu=1486.6 \mathrm{eV}) \mathrm{X}$-ray source (Kratos Analytical, Manchester, UK). Nicolet Magna 750 FTIR Spectrometer and Nic-Plan FTIR Microscope (Nicolet, USA) with pure $\mathrm{KBr}$ as the background was applied to record Fourier transform infrared (FTIR) spectra $\left(400-4000 \mathrm{~cm}^{-1}\right)$. The microgel samples for TEM, XPS and FTIR characterization were prepared using the procedure described below for generating AgNPs in the etalons with two modifications. First, to obtain sufficient sample for analysis we didn't wash the slide after microgel painting, which yielded a thick microgel layer for
AgNP generation. Second, the etalon's top Au layer was not deposited on the microgels, which allowed for their easy removal from the surface while avoiding potential contamination. Reflectance measurements were conducted using a USB2000+ spectrophotometer, an HL-2000-FHSA tungsten light source, and an R400-7-VISNIR optical fiber reflectance probe, all from Ocean Optics (Dunedin, FL). The spectra were recorded using Ocean Optics Spectra Suite spectroscopy software over a wavelength range of 400-1000 $\mathrm{nm}$.

\section{Synthesis of pNIPAm-co-AAc microgels}

pNIPAm-co-AAc microgels were synthesized via surfactant-free, free radical precipitation polymerization, according to an established protocol. ${ }^{22,29-33}$ The monomer, NIPAm (10.54 $\mathrm{mmol})$, and the crosslinker, BIS $(0.703 \mathrm{mmol})$, were fully dissolved in water $(99 \mathrm{~mL})$ with stirring in a beaker for $1 \mathrm{~h}$. The mixture was then filtered through a $0.2 \mu \mathrm{m}$ filter affixed to a $20 \mathrm{~mL}$ syringe into a $250 \mathrm{~mL}$, 3-necked round bottom flask. The flask was then equipped with a thermometer, a condenser/ $\mathrm{N}_{2}$ inlet/outlet, and a stir bar. The monomer solution was purged with $\mathrm{N}_{2}$ gas for $\sim 1 \mathrm{~h}$ while stirring and heating to $70{ }^{\circ} \mathrm{C}$. AAc $(2.812 \mathrm{mmol})$ and APS $(0.046 \mathrm{~g}$ in $1.0 \mathrm{~mL}$ water $)$ was then added to the pre-heated solution, respectively. The reaction continued for $4 \mathrm{~h}$. After cooling down, the turbid solution was filtered through glass wool to remove any large aggregates. The coagulum was rinsed and the collected liquid was diluted to 100 $\mathrm{mL}$. Aliquots of the microgel solution $(33 \mathrm{~mL})$ were centrifuged at a speed of 10000 relative centrifugal force (rcf) at $20{ }^{\circ} \mathrm{C}$ for $45 \mathrm{~min}$. The microgels were isolated and redispersed to their original volume $(\sim 33 \mathrm{~mL})$ with water. This centrifugation/ resuspension procedure was repeated 6 times. Finally, all of the centrifuged microgels were combined into one tube and diluted to $30 \mathrm{~mL}$ with water for storage.

\section{Etalon preparation}

Etalons were fabricated using the techniques that were detailed in previous studies. ${ }^{4}$ Typically, $25 \times 25 \mathrm{~mm}$ microscope coverslips (Fisher's Finest, Ottawa, ON) were washed with ethanol and dried with $\mathrm{N}_{2}$ gas and coated with $2 \mathrm{~nm} \mathrm{Cr}$ and then $15 \mathrm{~nm}$ $\mathrm{Au}$ via thermal evaporation at rates of 1 and $0.1 \AA \mathrm{s}^{-1}$, respectively (Torr International Inc., thermal evaporation system, Model THEUPG, New Windsor, NY). The Cr/Au coating was annealed in a Thermolyne muffle furnace at $250{ }^{\circ} \mathrm{C}$ for $3 \mathrm{~h}$ and allowed to cool. Then, aliquots $(40 \mu \mathrm{L})$ of concentrated microgels were added to the annealed Au-coated glass coverslips and spread out to coat the whole surface. The deposited microgels were dried on a hot plate set at $30^{\circ} \mathrm{C}$ for $30 \mathrm{~min}$. The excess free microgels not directly adhered to the Au layer were rinsed away with DI water. The samples were then soaked in DI water overnight at $30{ }^{\circ} \mathrm{C}$. The slides were then rinsed with DI water and dried with $\mathrm{N}_{2}$ gas. The top Au layer $(2 \mathrm{~nm} \mathrm{Cr}$ for adhesion, followed by $15 \mathrm{~nm} \mathrm{Au}$ ) was then deposited using thermal evaporation. The completed device was allowed to soak in DI water overnight before use. 


\section{AgNPs generation}

AgNPs were generated in the etalons by loading the microgels with $\mathrm{Ag}^{+}$followed by their in situ reduction with $\mathrm{NaBH}_{4} \cdot{ }^{34-37}$ Briefly, a $10 \mathrm{~mm}^{2}$ etalon was cut from the as-prepared larger etalon $(25 \times 25 \mathrm{~mm})$ and was subsequently immersed in $20 \mathrm{~mL}$ of DI water. The solution $\mathrm{pH}$ was then adjusted to 8.5 with $10 \mathrm{mM} \mathrm{NaOH}$ and the etalon was allowed to incubate overnight. The etalons were then added to a $10 \mathrm{~mL}$ solution containing $10 \mathrm{mM} \mathrm{AgNO}$ prepared with DI water for $24 \mathrm{~h}$ to allow for ionexchange between $\mathrm{Na}^{+}$and $\mathrm{Ag}^{+}$. Then, the etalons were immersed in $20 \mathrm{~mL}$ of DI water overnight to remove free $\mathrm{Ag}^{+}$. The $\mathrm{Ag}^{+}$-loaded etalons were then soaked in $10 \mathrm{~mL}$ of an aqueous solution of $\mathrm{NaBH}_{4}(10 \mathrm{mM})$ for $30 \mathrm{~min}$ in an ice water bath. Finally, the AgNP-loaded etalons were immersed in DI water and stored at $4{ }^{\circ} \mathrm{C}$.

\section{$\mathrm{H}_{2} \mathrm{O}_{2}$ sensing}

The optical properties of the devices can be predicted by eqn (1):

$$
m \lambda=2 n d \cos \theta
$$

where $n$ is the refractive index of the dielectric layer, $d$ is the distance between the etalon's two Au layers, $\theta$ is the angle of incident light relative to the device normal, and $m$ (an integer) is the order of the reflected peak.

The etalons were fixed in a specially designed stainless steel sample chamber and immersed in $30 \mathrm{~mL} \mathrm{pH} 7.0$ phosphate buffer solution ( $5 \mathrm{mM}$ ) at room temperature. The chamber was sealed with a metal cover with a port for $\mathrm{H}_{2} \mathrm{O}_{2}$ addition $(30 \% \mathrm{w} /$ $\mathrm{w}, 10 \mathrm{M}$ ) could be introduced to the solution. The center of the cover had a hole where the reflectance probe could be secured and exposed to the etalon. The light source's intensity and distance from the etalon was adjusted to result in the highest quality reflectance spectra (well defined peaks with high intensity). Spectra were recorded until they stabilized then more $\mathrm{H}_{2} \mathrm{O}_{2}$ was added to solution. Each experiment was repeated at least three times. To regenerate AgNPs in the etalons, a similar process to the original AgNPs generation was used. Briefly, the used etalons were immersed in $20 \mathrm{~mL}$ of DI water adjusted to 8.5 with $10 \mathrm{mM} \mathrm{NaOH}$ and allowed to incubate overnight. The etalons were then added to a $10 \mathrm{~mL}$ aqueous solution containing $10 \mathrm{mM} \mathrm{AgNO}_{3}$ for $24 \mathrm{~h}$ and then in DI water overnight. The $\mathrm{Ag}^{+}$-reloaded etalons were then soaked in $10 \mathrm{~mL}$ of an aqueous solution of $\mathrm{NaBH}_{4}(10 \mathrm{mM})$ for $30 \mathrm{~min}$ in an ice water bath. Finally, the recharged AgNP-loaded etalons were prepared and stored in DI water at $4{ }^{\circ} \mathrm{C}$.

\section{Results and discussion}

The synthetic route for generating AgNPs inside pNIPAm-co-AAc microgel-based etalon is shown schematically in Scheme 1. The solution $\mathrm{pH}$ for loading $\mathrm{Ag}^{+}$was 8.5 , according to our previous report. $^{22}$ The pNIPAm-co-AAc microgels were composed of $\mathrm{COOH}$ groups that could be deprotonated by increasing the solution $\mathrm{pH}$ above the $\mathrm{p} K_{\mathrm{a}}$ for AAc (4.25), which rendered them negatively charged. The negative charges in the pNIPAm-co-AAc microgels could increase the interaction strength between the microgels and the $\mathrm{Ag}^{+}$, thus leading to enrichment in the microgels. As detailed in the Experimental section above, the microgels in the etalons were then soaked in DI water to remove any unbound $\mathrm{Ag}^{+}$, and the $\mathrm{Ag}^{+}$-loaded etalon subsequently exposed to $\mathrm{NaBH}_{4}$ to form AgNPs. Of note, the in situ reduction of $\mathrm{Ag}^{+}$was performed at low temperature by immersing the reaction vessel into an ice-water bath, which largely prevents the liberation of hydrogen gas. ${ }^{38}$ We found that the AgNP synthesis at room temperature generates hydrogen gas bubbles, due to the rapid hydrolysis of $\mathrm{NaBH}_{4}$, which leads to the destruction of the etalon's top Au layer, and a loss of the etalon's optical properties (Fig. S1†). As can be seen in Fig. 1A, the etalon was undamaged using the low temperature AgNP synthesis. We note that because the etalons were undamaged by this route to generating AgNPs, the AgNPs could be regenerated multiple times in the etalon for reuse. Furthermore, the AgNP synthesis at low temperature was found to be effective at narrowing the size distribution of nanoparticles, compared to what was reported previously. ${ }^{39-42}$ In this study, the as-synthesized AgNPs possess a rather uniform size (Fig. 1B) ranging from 5 to $7 \mathrm{~nm}$ (Fig. 1C) and no large diameter particles were observed, as evidenced in the representative TEM image of the hybrid microgels (Fig. 1D). Furthermore, as can be seen in Fig. S2, $\dagger$ characteristic binding energy peaks of $\mathrm{Ag} 3 \mathrm{~d}_{5 / 2}$ and $\mathrm{Ag} 3 \mathrm{~d}_{3 / 2}$ appeared at 367.9 and $373.9 \mathrm{eV}$, respectively, after the incorporation of the AgNPs. Such binding energies are between $\mathrm{Ag}^{0}$ $\left(3 \mathrm{~d}_{5 / 2}: 368.2 \mathrm{eV}\right.$ and $3 \mathrm{~d}_{3 / 2}: 374.2 \mathrm{eV}$, respectively) and $\mathrm{Ag}^{+}\left(3 \mathrm{~d}_{5 / 2}\right.$ : $367.5 \mathrm{eV}$ and $3 \mathrm{~d}_{3 / 2}: 373.5 \mathrm{eV}$, respectively). The shift to lower binding energies for the AgNPs can be attributed to the interaction between oxygen and silver particles. ${ }^{43}$ These observations indicate the role of the microgels as microreactors, where the coordinated $\mathrm{Ag}^{+}$could be steady reduced to AgNPs by $\mathrm{NaBH}_{4}$ in the microgel network.

The role of the pNIPAm-co-AAc microgels as ligands to AgNPs was then investigated by FTIR spectroscopy. As shown in Fig. 2, the pure pNIPAm-co-AAc microgels exhibited a series of bands centered at $3306 \mathrm{~cm}^{-1}, 1715 \mathrm{~cm}^{-1}, 1649 \mathrm{~cm}^{-1}$ and $1541 \mathrm{~cm}^{-1}$, which were attributed to the $\mathrm{N}-\mathrm{H}$ stretching, ${ }^{44}$ free carboxyl group $(-\mathrm{COOH}),{ }^{45}$ the amide carbonyl I bond, ${ }^{46}$ and the amide carbonyl II bond, ${ }^{47}$ respectively, in accordance with the characteristic IR absorption of the pNIPAm-co-AAc polymers. ${ }^{41}$ The incorporation of AgNPs to form the hybrid microgels was shown to lead to three major changes, as shown in Fig. 2. First, the $\mathrm{N}-\mathrm{H}$ stretching peak $\left(3306 \mathrm{~cm}^{-1}\right)$ was shifted to $3300 \mathrm{~cm}^{-1}$; second, the free $-\mathrm{COOH}$ group band peak at $1715 \mathrm{~cm}^{-1}$ disappeared; and third, the amide carbonyl band (I: $1649 \mathrm{~cm}^{-1}$ and II: $1541 \mathrm{~cm}^{-1}$ ) was shifted to $1646 \mathrm{~cm}^{-1}$ and $1550 \mathrm{~cm}^{-1}$, respectively, indicating the binding interaction between AgNPs and the polymer network. This was also supported by previous reports that the coordination between metal cations and nitrogen species of pNIPAm-based microgels could lead to a shift of the carbonyl bands. ${ }^{48}$

Since the microgels have a high affinity for the AgNPs, we hypothesize that they act as a microgel crosslinker. That is, the binding interaction of microgels with AgNPs generates a contractive force, rendering the microgels deswollen; 


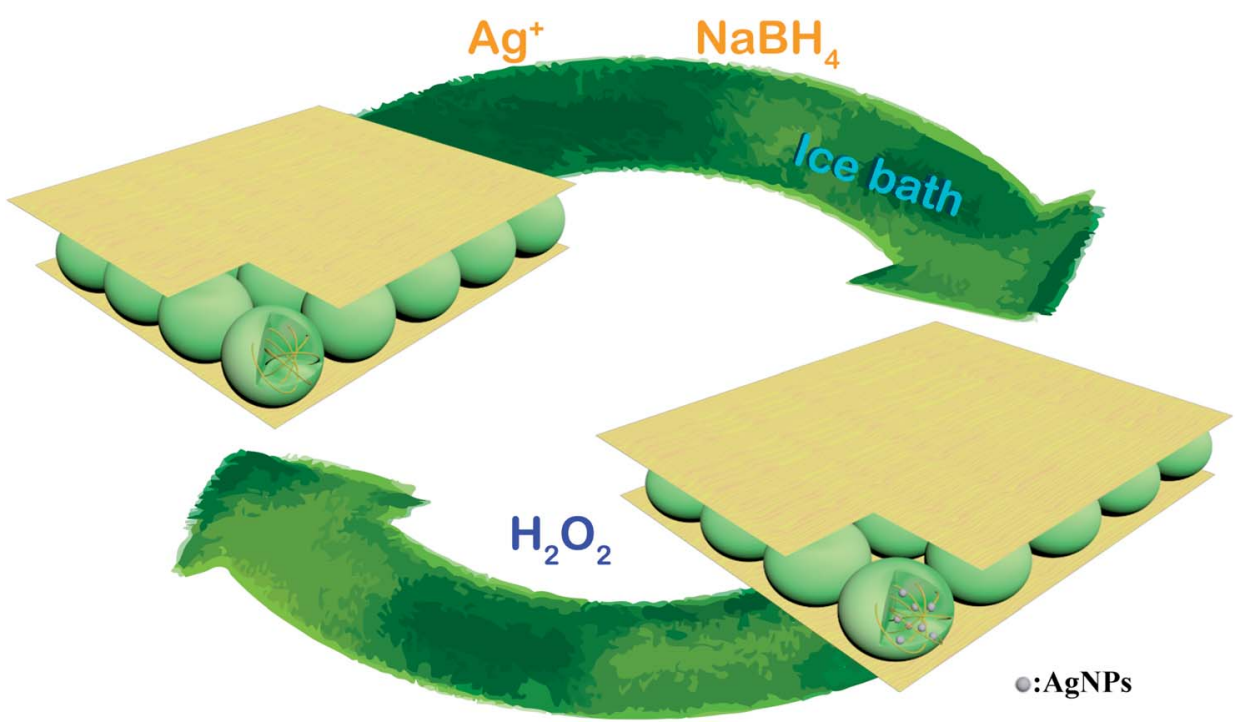

Scheme 1 Schematic illustration of the in situ fabrication method of AgNPs@pNIPAm-co-AAc hybrid microgels-based etalon and response mechanism of the etalon.

therefore, the microgels should swell upon removal of the AgNPs. As a result, we expect the removal of AgNPs from microgels in the etalons to yield a red-shift in the etalon's reflectance peaks, as predicted from eqn (1). AgNPs are known to decompose in the presence of $\mathrm{H}_{2} \mathrm{O}_{2}$ through oxidation. ${ }^{22}$ The proposed decomposition mechanism is showed as follows: ${ }^{22}$

$$
\mathrm{Ag}_{n}+\mathrm{H}_{2} \mathrm{O}_{2}=\mathrm{Ag}_{n} / \mathrm{HOOH}
$$

A)

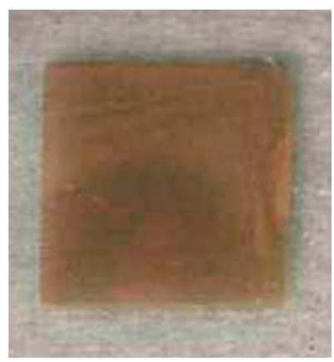

C)

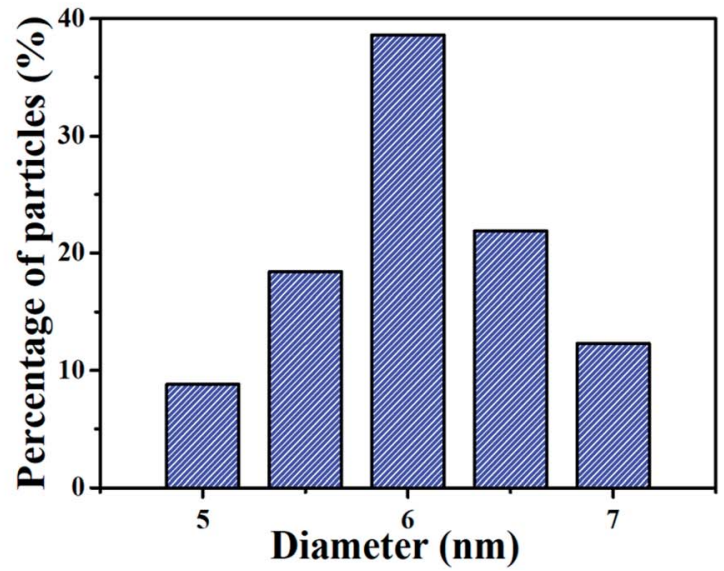

B)

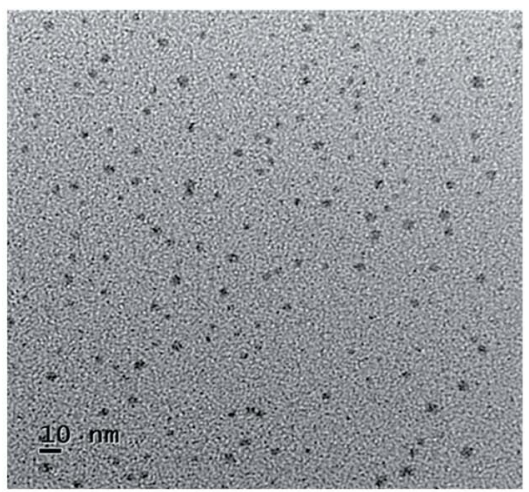

D)

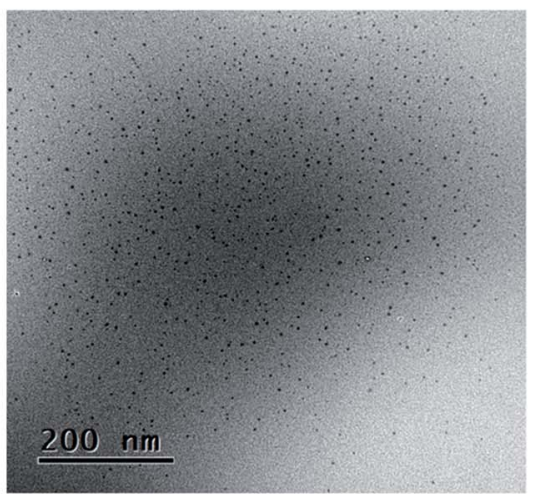

Fig. 1 (A) Photograph of the AgNP-loaded etalon in solution at pH 7.0. (B) TEM image of AgNPs within microgels and (C) the corresponding histogram of size distribution of AgNPs. (D) TEM image of the AgNPs@pNIPAm-co-AAc hybrid microgels which were collected from an etalon prepared without the top gold layer (see Experimental section). 


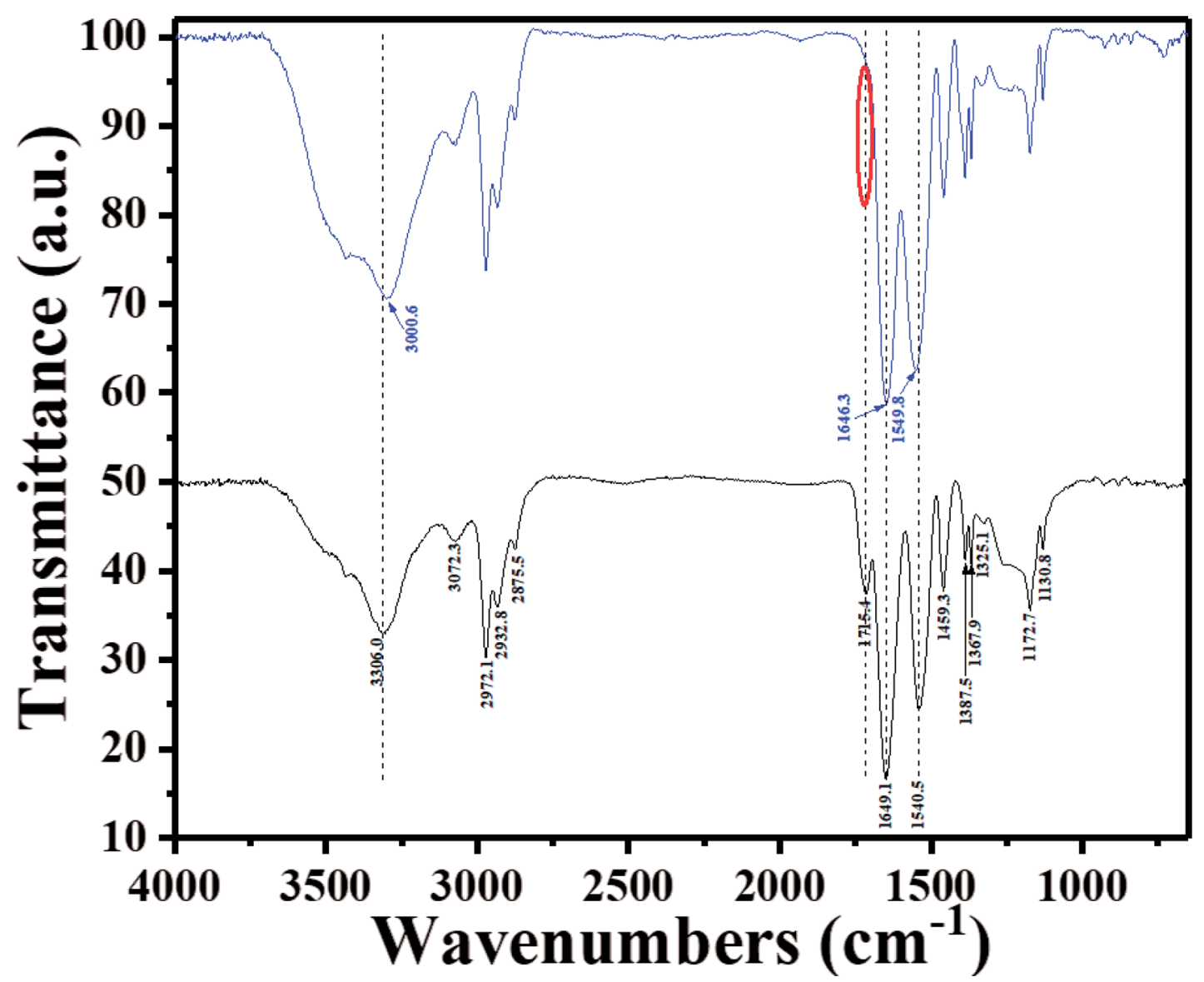

Fig. 2 FT-IR spectra of the blank microgels (black) and the AgNPs@pNIPAm-co-AAc hybrid microgels (blue). The red circle shows the band where the free carboxyl group is.

$$
\begin{gathered}
\mathrm{Ag}_{n} / \mathrm{HOOH}=\mathrm{Ag}_{n} / \mathrm{HOO}^{\bullet} \\
2 \mathrm{Ag}_{n} / \mathrm{HOO}^{*}=2 \mathrm{Ag}_{n}+2 \mathrm{HO}^{\bullet}+\mathrm{O}_{2} \\
\mathrm{Ag}_{n}+\mathrm{HO}^{\bullet}=\mathrm{Ag}_{n-1} /\left[\mathrm{Ag}^{+}\right]+\mathrm{H}_{2} \mathrm{O}
\end{gathered}
$$

We thus hypothesize that the introduction of $\mathrm{H}_{2} \mathrm{O}_{2}$ can trigger a red-shift of the reflectance peaks from the AgNP-loaded etalons. As can be seen in Fig. 3A, a characteristic multipeak reflectance spectrum of the AgNP-etalon was observed and it shows three reflectance peaks at $475 \mathrm{~nm}, 635 \mathrm{~nm}$ and $910 \mathrm{~nm}$. The most significant peak (i.e., $636 \mathrm{~nm}$ ) was selected in the following experiments for analysis. It was observed that the peak red-shifted $24 \mathrm{~nm}$ in the presence of $10 \mathrm{mM} \mathrm{H}_{2} \mathrm{O}_{2}$, while no changes were observed by adding the same amount of $\mathrm{H}_{2} \mathrm{O}_{2}$ to an etalon without AgNPs (Fig. 3B), indicating that the shift could be a result of the presence of the AgNPs. Furthermore, we observed a change in the intensity of the reflectance peaks in response to $\mathrm{H}_{2} \mathrm{O}_{2}$, which is not typically observed from the microgel-based etalons. ${ }^{4,49}$ AgNPs are also well known to absorb visible light due to the excitation of plasmons. ${ }^{50}$ With the etalons here, we also found that the intensity of reflected light increased from $75 \%$ to $120 \%$, possibly due to the reduction of AgNP light absorption. Thus, more photons could make it back to the reflectance probe, as opposed to being absorbed by the AgNPs. In addition, the intensity of the peak valley $(\sim 25 \%)$, almost remained unchanged. This increase of the intensity ratio of peak to valley improved contrast, allowing for facile visual characterization. Specifically, the addition of $\mathrm{H}_{2} \mathrm{O}_{2}$ led to the color change of the AgNP-etalon from brassy yellow to green (Fig. 3A inset). Therefore, sensing $\mathrm{H}_{2} \mathrm{O}_{2}$ by monitoring two parameters, i.e., red-shifted reflectance peak and increased intensity, can be attributed to the swelling of the microgel layer and the reduction of the AgNP absorption, respectively, as a result of decomposition of the loaded AgNPs. The $\mathrm{H}_{2} \mathrm{O}_{2}$ sensing mechanism is depicted in Scheme 2.

The sensing performance of this device was further investigated by exposing it to $10 \mathrm{mM} \mathrm{H}_{2} \mathrm{O}_{2}$. Fig. 3C shows that the response of the AgNP-etalon to $\mathrm{H}_{2} \mathrm{O}_{2}$ was complete in $\sim 1 \mathrm{~h}$. We note that the responsivity of this device towards $\mathrm{H}_{2} \mathrm{O}_{2}$ was enhanced (Fig. 3D) in pH 3.0 buffer solution, possibly due to the reduction of electrostatic repulsions of protonated intramicrogels ( $K_{\mathrm{a}}$ of AAc: 4.25 ), which suppressed the microgel deswelling. Next, we determined the ability of the AgNP-loaded etalons to quantify $\mathrm{H}_{2} \mathrm{O}_{2}$ concentration in solution. As can be seen in Fig. 4A, as the concentration of $\mathrm{H}_{2} \mathrm{O}_{2}$ increased from $0.5 \mathrm{mM}$ to $10 \mathrm{mM}$, the selected reflectance peak of the AgNPetalon correspondingly red-shifted, exhibiting a good linear 

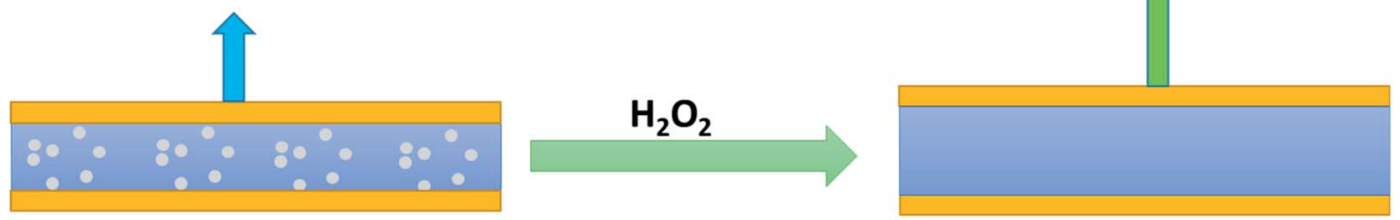

AgNPs

Microgel layer

\section{Reflected hv}

Scheme 2 Schematic depiction of the $\mathrm{H}_{2} \mathrm{O}_{2}$ sensing process. (Left), light of a specific wavelength is reflected, while a different wavelength with increased intensity (indicated by the longer arrow) is reflected after the AgNPs are degraded upon $\mathrm{H}_{2} \mathrm{O}_{2}$ addition.

relationship within the concentration ranging from $0.5 \mathrm{mM}$ to $6 \mathrm{mM}\left(R^{2}=0.9943\right)$ (Fig. $\left.4 \mathrm{~B}\right)$, which is comparable to previous methods of $\mathrm{H}_{2} \mathrm{O}_{2}$ detection (Table S1 $\dagger$ ). Also, the reflectance intensity of this device increased in response to escalated concentrations of $\mathrm{H}_{2} \mathrm{O}_{2}$ (Fig. 4C).

As the exposure of the etalons to $\mathrm{H}_{2} \mathrm{O}_{2}$ caused the dissolution of the AgNPs, their regeneration in the etalons was also investigated. Specifically, addition of $5 \mathrm{mM} \mathrm{H}_{2} \mathrm{O}_{2}$ caused $16 \mathrm{~nm}$ red-shift of the reflectance peak of the device (Fig. $4 \mathrm{~A}$ and $\mathrm{D}$ ) and a dramatic decrease in the amount of AgNPs in the microgels, as revealed by the representative TEM images in Fig. S3.† Due to the etalon structure remaining intact, we reason that AgNPs could be regenerated in the microgels and the etalons reused. To show this, we reloaded the microgels with $\mathrm{Ag}^{+}$and carried
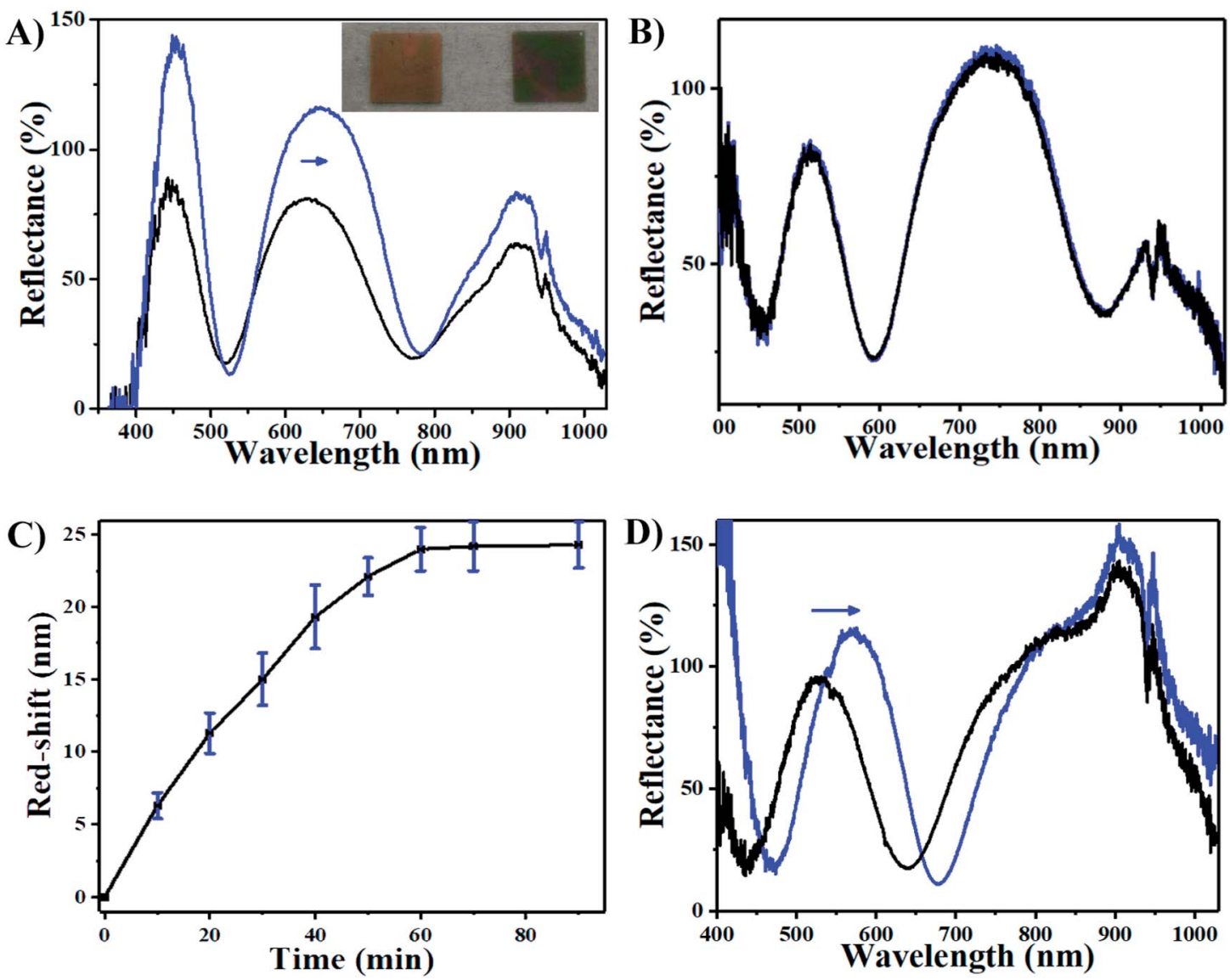

Fig. 3 Reflectance spectra of etalon (A) with and (B) without AgNPs upon exposure to $10 \mathrm{mM} \mathrm{H}_{2} \mathrm{O}_{2}$ at pH 7.0. The inserted photograph in (A) shows the AgNPs containing etalon before and after adding $10 \mathrm{mM} \mathrm{H}_{2} \mathrm{O}_{2}$ from left to right, respectively. (C) Peak shifts as a function of reaction time after addition of $10 \mathrm{mM} \mathrm{H}_{2} \mathrm{O}_{2}$ at $\mathrm{pH}$ 7.0. Data are mean values \pm standard deviation of three independent experiments. (D) Reflectance spectra of etalon with AgNPs upon exposure to $10 \mathrm{mM} \mathrm{H}_{2} \mathrm{O}_{2}$ at $\mathrm{pH}$ 3.0. The blue arrows show the direction of peak shift. 

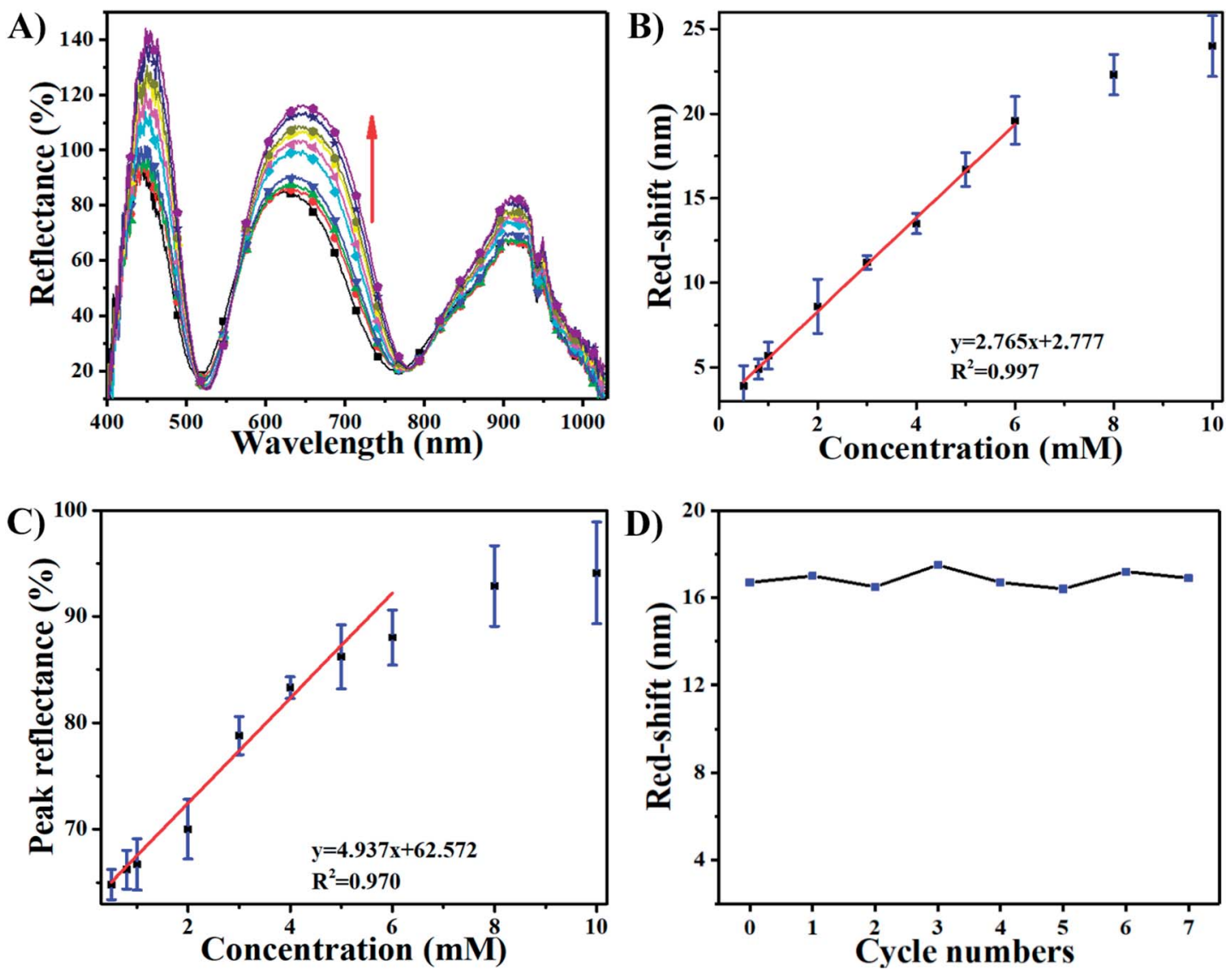

Fig. 4 (A) Reflectance spectra of AgNPs-loaded etalon upon exposure to various concentrations of $\mathrm{H}_{2} \mathrm{O}_{2}$ at $\mathrm{pH} 7.0$ from 0.5 (bottom) to 10 mM (top); the direction of the peak shift is indicated by the red arrow. (B) Peak shifts as a function of $\mathrm{H}_{2} \mathrm{O}_{2}$ concentration. (C) Peak reflectance as a function of $\mathrm{H}_{2} \mathrm{O}_{2}$ concentration. (D) The cycles of the red shifts of etalon though the "consuming-recharging" of $\mathrm{AgNPs}$ at $\mathrm{pH} 7.0\left(\mathrm{H}_{2} \mathrm{O}_{2}\right.$ concentration: $5 \mathrm{mM}$ ). Data are mean \pm standard deviation of three independent experiments.

out the $\mathrm{NaBH}_{4}$ reduction, as described in Experiment section above. After incorporating AgNPs in the etalon again, a similar response of the etalon to $\mathrm{H}_{2} \mathrm{O}_{2}$ can be fully recovered, as can be seen in Fig. 4D. Furthermore, the AgNP regeneration could be repeated at least 7 times without losing the performance efficiency of this device, as shown in Fig. 4D.

\section{Conclusion}

In summary, we reported an approach for the in situ generation of AgNPs inside pNIPAm-co-AAc microgels that were the responsive component of an etalon. We characterized the synthesized AgNPs-loaded microgels within the etalon, and demonstrated that the incorporation of the AgNPs allowed the etalons to respond to $\mathrm{H}_{2} \mathrm{O}_{2}$ in solution by changing the wavelength of the reflected light and the reflected light intensity. We attributed this dual response towards $\mathrm{H}_{2} \mathrm{O}_{2}$ to the oxidative decomposition of the AgNPs within the microgels, enabling the microgels to swell and the light that was absorbed by the AgNPs to be reflected. Finally, we demonstrated that the functional etalons could be reused multiple times through repeatedly "consuming and recharging" the AgNPs. While this study was only done with $\mathrm{H}_{2} \mathrm{O}_{2}$ as a proof of concept, it is a promising approach to extend the versatility of etalons to sense species of broad relevance simply by modification with nanomaterials, enriching the polymer-based photonic sensor portfolio. This work has also demonstrated that the dual signal sensing can be a potential route to improved sensor performance, i.e., enhanced selectivity and accuracy.

\section{Conflicts of interest}

There are no conflicts to declare.

\section{Acknowledgements}

MJS acknowledges funding from the University of Alberta (the Department of Chemistry and the Faculty of Science), the Natural Sciences and Engineering Research Council of Canada (NSERC), the Canada Foundation for Innovation (CFI), the Alberta Advanced Education \& Technology Small Equipment Grants Program (AET/SEGP), Grand Challenges Canada and ICIMPACTS. XZ and TS acknowledge support from the National Natural Science Foundation of China (Grant No. 21727815), the Beijing Municipal Science and Technology Commission (z131102002813058), Beijing Natural Science Foundation 
(2184107) and the Fundamental Research Funds for the Central Universities (FRF-TP-15-096A1). MJS, XZ and TS acknowledge the Research Fund for International Young Scientists of the National Natural Science Foundation of China (21750110449).

\section{References}

1 J. M. Weissman, H. B. Sunkara, A. S. Tse and S. A. Asher, Science, 1996, 274, 959-963.

2 J. H. Holtz and S. A. Asher, Nature, 1997, 389, 829.

3 C. Fenzl, T. Hirsch and O. S. Wolfbeis, Angew. Chem., Int. Ed., 2014, 53, 3318-3335.

4 Q. M. Zhang, D. Berg, J. Duan, S. M. Mugo and M. J. Serpe, ACS Appl. Mater. Interfaces, 2016, 8, 27264-27269.

5 C. D. Sorrell and M. J. Serpe, Adv. Mater., 2011, 23, 40884092.

6 C. D. Sorrell, M. C. D. Carter and M. J. Serpe, Adv. Funct. Mater., 2011, 21, 425-433.

7 L. Hu and M. J. Serpe, Chem. Commun., 2013, 49, 2649-2651. 8 Q. M. Zhang, W. Wang, Y.-Q. Su, E. J. M. Hensen and M. J. Serpe, Chem. Mater., 2016, 28, 259-265.

9 Q. M. Zhang, A. Ahiabu, Y. Gao and M. J. Serpe, J. Mater. Chem. C, 2015, 3, 495-498.

10 M. R. Islam and M. J. Serpe, Biosens. Bioelectron., 2013, 49, 133-138.

11 M. R. Islam and M. J. Serpe, Anal. Chim. Acta, 2014, 843, 8388.

12 Q. M. Zhang, X. Li, M. R. Islam, M. Wei and M. J. Serpe, J. Mater. Chem. C, 2014, 2, 6961-6965.

13 T. Shu, J. Wang, L. Su and X. Zhang, Anal. Chem., 2016, 88, 11193-11198.

14 T. Shu, L. Su, J. Wang, C. Li and X. Zhang, Biosens. Bioelectron., 2015, 66, 155-161.

15 M. Holzinger, A. Le Goff and S. Cosnier, Front. Chem., 2014, 2, 63.

16 R. M. Kong, X. B. Zhang, Z. Chen and W. H. Tan, Small, 2011, 7, 2428-2436.

17 P. D. Howes, R. Chandrawati and M. M. Stevens, Science, 2014, 346, 1247390.

18 T. Shu, B. W. Gao, H. K. Yang, L. Su and X. J. Zhang, Curr. Nanosci., 2016, 12, 405-410.

19 T. Shu, J. X. Wang, X. Q. Li, X. J. Zhang and L. Su, Curr. Nanosci., 2015, 11, 702-709.

20 R. Begum, Z. H. Farooqi and S. R. Khan, Int. J. Polym. Mater. Polym. Biomater., 2016, 65, 841-852.

21 S. Shi, Q. Wang, T. Wang, S. Ren, Y. Gao and N. Wang, J. Phys. Chem. B, 2014, 118, 7177-7186.

22 D. M. Han, Q. M. Zhang and M. J. Serpe, Nanoscale, 2015, 7, 2784-2789.

23 D. K. Gautam, M. M. Misro, S. P. Chaki and N. Sehgal, Apoptosis, 2006, 11, 39-46.

24 C. R. Reczek and N. S. Chandel, Curr. Opin. Cell Biol., 2015, 33, 8-13.

25 W. Droge and H. M. Schipper, Aging Cell, 2007, 6, 361-370.

26 S. DiMauro and E. A. Schon, Annu. Rev. Neurosci., 2008, 31, 91-123.
27 R. C. Yin, D. P. Zhang, Y. L. Song, B. Z. Zhu and H. L. Wang, Sci. Rep., 2013, 3, 1269.

28 T. Finkel, M. Serrano and M. A. Blasco, Nature, 2007, 448, 767-774.

29 Z. H. Farooqi, S. R. Khan, T. Hussain, R. Begum, K. Ejaz, S. Majeed, M. Ajmal, F. Kanwal and M. Siddiq, Korean J. Chem. Eng., 2014, 31, 1674-1680.

30 Z. H. Farooqi, S. Iqbal, S. R. Khan, F. Kanwal and R. Begum, e-Polym., 2014, 14, 313-321.

31 Z. H. Farooqi, T. Sakhawat, S. R. Khan, F. Kanwal, M. Usman and R. Begum, Mater. Sci.-Pol., 2015, 33, 185-192.

32 K. C. Clarke, S. N. Dunham and L. A. Lyon, Chem. Mater., 2015, 27, 1391-1396.

33 H. Bachman, A. C. Brown, K. C. Clarke, K. S. Dhada, A. Douglas, C. E. Hansen, E. Herman, J. S. Hyatt, P. Kodlekere, Z. Y. Meng, S. Saxena, M. W. Spears, N. Welsch and L. A. Lyon, Soft Matter, 2015, 11, 2018-2028. 34 Z. H. Farooqi, R. Khalid, R. Begum, U. Farooq, Q. Wu, W. Wu, M. Ajmal, A. Irfan and K. Naseem, Environ. Technol., 2018, 1-10, DOI: 10.1080/09593330.2018.1435737.

35 M. Shahid, Z. H. Farooqi, R. Begum, K. Naseem, M. Ajmal and A. Irfan, Korean J. Chem. Eng., 2018, DOI: 10.1007/ s11814-018-0016-x.

36 R. Begum, Z. H. Farooqi, Z. Butt, Q. Wu, W. Wu and A. Irfan, J. Environ. Sci., 2017, DOI: 10.1016/j.jes.2017.12.003.

37 R. Begum, Z. H. Farooqi, E. Ahmed, K. Naseem, S. Ashraf, A. Sharif and R. Rehan, Appl. Organomet. Chem., 2017, 31, e3563.

38 Y. H. Yan, H. Y. Guan, S. Liu and R. Y. Jiang, Ceram. Int., 2014, 40, 9095-9100.

39 Y. Dong, Y. Ma, T. Zhai, F. Shen, Y. Zeng, H. Fu and J. Yao, Macromol. Rapid Commun., 2007, 28, 2339-2345.

40 X. Liu, C. Zhang, J. Yang, D. Lin, L. Zhang, X. Chen and L. Zha, RSC Adv., 2013, 3, 3384-3390.

41 A. Khan, A. M. El-Toni, S. Alrokayan, M. Alsalhi, M. Alhoshan and A. S. Aldwayyan, Colloids Surf., A, 2011, 377, 356-360.

42 S. Agnihotri, S. Mukherji and S. Mukherji, Appl. Nanosci., 2012, 2, 179-188.

43 Y. Zhao, Y. Zhou, X. Wu, L. Wang, L. Xu and S. Wei, Appl. Surf. Sci., 2012, 258, 8867-8873.

44 B. Akgun, E. Savci and D. Avci, J. Polym. Sci., Part A: Polym. Chem., 2012, 50, 801-810.

45 L. Q. Zhu, C. Z. Zhao, J. W. Zhang and D. R. Gong, RSC Adv., 2015, 5, 84263-84268.

46 Y. L. Liu, L. J. Xing, Q. S. Zhang, Q. F. Mu, P. F. Liu, K. Chen, L. Chen, X. Y. Zhang, K. Wang and Y. Wei, Colloid Polym. Sci., 2016, 294, 617-628.

47 A. R. Karimi, F. Azadikhah, L. Rahimi and S. Ghadimi, Colloids Surf., A, 2015, 484, 304-312.

48 H. G. Becker and G. Schmidt-Naake, J. Appl. Polym. Sci., 2003, 90, 2322-2332.

49 W. Zhang, M. Wei, W. S. P. Carvalho and M. J. Serpe, Anal. Chim. Acta, 2018, 999, 139-143.

50 A. D. McFarland and R. P. Van Duyne, Nano Lett., 2003, 3, 1057-1062. 